NASA Technical Memorandum 110281

U. S. Army Research Laboratory Technical Report 1226

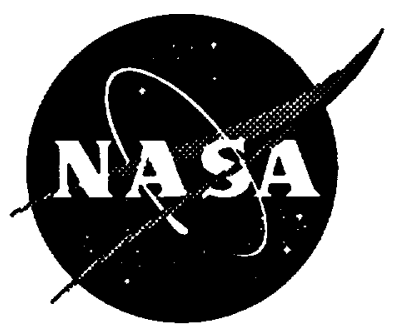

\title{
Performance of Optimized Actuator and Sensor Arrays in an Active Noise Control System
}

D. L. Palumbo and S. L. Padula

Langley Research Center, Hampton, Virginia

K. H. Lyle and J. H. Cline

Vehicle Structures Directorate

U.S. Army Research Laboratory

Langley Research Center, Hampton, Virginia

R. H. Cabell

Virginia Polytechnic Institute and State University, Blacksburg, Virginia

September 1996

National Aeronautics and

Space Administration

Langley Research Center

Hampton, Virginia 23681-0001 


\title{
PERFORMANCE OF OPTIMIZED ACTUATOR AND SENSOR ARRAYS IN AN ACTIVE NOISE CONTROL SYSTEM
}

\author{
D. L. Palumbo and S. L. Padula \\ NASA Langley Research Center, Hampton, VA \\ K. H. Lyle and J. H. Cline \\ U. S. Army Vehicle Structures Directorate-ARL, Hampton, VA \\ R. H. Cabell \\ Virginia Polytechnic Institute and State University \\ Blacksburg, VA
}

\begin{abstract}
Experiments have been conducted in NASA Langley's Acoustics and Dynamics Laboratory to determine the effectiveness of optimized actuator/sensor architectures and controller algorithms for active control of harmonic interior noise. Tests were conducted in a large scale fuselage model - a composite cylinder which simulates a commuter class aircraft fuselage with three sections of trim panel and a floor. Using an optimization technique based on the component transfer functions, combinations of 4 out of 8 piezoceramic actuators and 8 out of 462 microphone locations were evaluated against predicted performance. A combinatorial optimization technique call tabu search was employed to select the optimum transducer arrays. Three test frequencies represent the cases of a strong acoustic and strong structural response, a weak acoustic and strong structural response and a strong acoustic and weak structural response. Noise reduction was obtained using a Time Averaged/Gradient Descent (TAGD) controller. Results indicate that the optimization technique successfully predicted best and worst case performance. An enhancement of the TAGD control algorithm was also evaluated. The principal components of the actuator/sensor transfer functions were used in the PC-TAGD controller. The principal components are shown to be independent of each other while providing control as effective as the standard TAGD.
\end{abstract}

\section{Introduction}

This work is part of a continuing effort at NASA's Langley Research Center to better understand the methods and techniques that can be brought to bear on the problem of active control of aircraft interior noise. A primary concern in this effort has been harmonic noise of the type that is created by propeller blades and other rotating machinery. Of specific interest in this paper is the integration of arrays of actuators and sensors into an effective active structural acoustic control (ASAC) system.

Initial experiments and analytical studies using ASAC to control interior noise focused on actuation of the primary shell of the structure ${ }^{1,2}$. These efforts demonstrated that control sufficient to achieve substantial reduction $(\sim 12 \mathrm{~dB})$ in interior noise levels could be achieved. However, concerns have been raised in the aircraft industry over any additional structural fatigue that an ASAC system might introduce. If the ASAC system did indeed cause increased structural fatigue, then the cost of certifying and maintaining the aircraft would increase, thereby compromising any cost advantages that ASAC may provide.

An alternative approach has been investigated which uses force actuators bonded to the trim panels ${ }^{3}$. In this previous effort, several piezoelectric actuators were bonded to the surface of a trim panel which was hard mounted to the ring frames of a composite fuselage model. Modal decomposition of the acoustic field was used to select actuator/sensor sets that were well matched to the primary response (excited by an external acoustic source). Best results were obtained when the actuator modal response did indeed match the primary, but even then only moderate noise reduction was possible $(\sim 5 \mathrm{~dB})$.

This paper reports efforts to improve the effectiveness of trim panel ASAC by using optimization techniques to select best case actuator/sensor architectures and controller algorithms. The performance of the actuator/sensor arrays were 
tested using a Time Average/Gradient Descent controller (TAGD). To better judge how well the optimization procedure predicted the system response, worst case actuator/sensor arrays were also computed and tested. These results are compared to results obtained with actuator/sensor arrays chosen using modal decomposition procedures ${ }^{3}$. Finally, a modified version of the TAGD controller was tested in which the principal components of the actuator/sensor transfer functions were used in the gradient descent algorithm. The benefit of this approach is that the principal components with their corresponding error components are independent of each other. The result is a control algorithm in which each channel of control is orthogonal to every other channel. This provides more robust convergence during adaptation, and enables parallel implementation of the algorithm.

The next sections provide an overview of the optimization techniques. This is followed by a description of the experiment procedure. The paper concludes with the details of implementing the optimization techniques and the associated results.

\section{Architecture Optimization Overview}

Figure 1 is a block diagram of a generic noise control problem. It is desired to reduce the acoustic noise field, $e_{j}$, which is produced by the primary source, $p$, by applying control through the actuator array, $c_{k}$. The coupling of the primary and control sources with the acoustic field is given by their respective transfer functions, $\mathrm{H}^{p}$ and $\mathrm{H}^{c}$. The relationship of these components is written as

$$
e_{j}=\sum_{k=1}^{N_{c}} H_{k}^{c} c_{k}+H^{p} p,
$$

where $N_{c}$ is the number of actuators in the control array. A measure of the total sound field of a particular control solution, $J_{r}$, is the sum of the squared pressures over the $N_{r}$ points which define the response field.

$$
J_{r}=\sum_{j=1}^{N_{r}} e_{j} e_{j}^{*},
$$

where ${ }^{*}$ is the complex conjugate. The control is most effective (the performance is optimized) when $J_{r}$ is minimized.

Given a wide choice of actuator and sensor locations, the goal of optimization is to identify a subset of the actuators and sensors which provides the best performance, i.e. has the potential to reduce the acoustic field to its lowest level. The optimization methodology

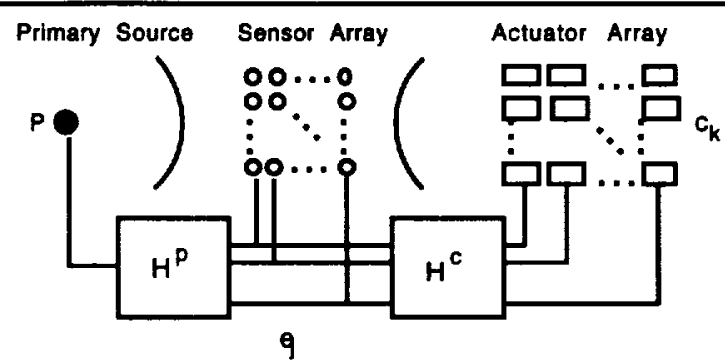

Figure 1. A generic noise control system

which was used is conceptually simple. From a given starting state, perturbations of sensors and actuator settings are simulated, those combinations achieving the best performance levels are retained and refined until a global optimum is found. As basis for the simulation, broad band transfer functions between the noise sources and the acoustic field are acquired.

At a frequency of interest, equation (1) is solved for the control output and the resulting response using a least squares method which minimizes $J .^{4}$ For a total number of possible actuators, $N_{a}$, there are $N_{a}$ combination $N_{c}$ possible subsets of control arrays. The combination operator is given by,

$$
\left(\frac{N_{a} !}{\left(N_{a}-N_{c}\right) !^{*} N_{c} !}\right) \text {. }
$$

An exhaustive search of all possible control array subsets would uncover one array at which the response is minimized globally. For the optimum control array, $c^{\circ}$, a corresponding optimum sensor array, $s^{\circ}$, must be found. Given $N_{s}$ elements of the sensor subset, there are $N_{r}$ combination $N_{s}$ possible subsets of the response field at which sensors may be located. Equations (1) and (2) become

$$
\begin{aligned}
& s_{l}=\sum_{k=1}^{N_{c}} H_{k}^{c} c_{k}^{o}+H^{p} p \\
& \text { and } \\
& J_{s}=\sum_{l=1}^{N_{s} s_{l} s_{l}^{*} .}
\end{aligned}
$$

A search similar to the one performed to locate $c^{0}$ is performed to find $s^{0}$. 
The method described above uses exhaustive search to find the optimum actuator and sensor subsets. For practical applications, this may not be possible.

Consider a test matrix for the actuators on a cylindrical shell where actuators are placed every $10^{\circ}$ from $-90^{\circ}$ to $0^{\circ}$ along the circumference and every $1 / 3$ meter over 3 meters along the axis for a total of 100 possible actuator locations. If the best 10 actuators sites are to be selected, then over $10^{13}$ combinations would need to be considered. Clearly an alternative search procedure is required.

The search space can be narrowed using techniques of integer and combinatorial optimization ${ }^{5}$. These techniques rely on the evaluation of a cost for each state. Having this information, the search through the state space can be directed along those paths which promise to reduce the cost the most. The expressions for $J_{r}$ and $J_{s}$ in equations (2) and (5) respectively are ideal cost metrics. One drawback of combinatorial optimization is that the search may converge to a local minimum, i.e., a state in which the cost is not the global minimum, yet, each step to a nearby state increases the cost. Combinatorial searches may also visit the same state repeatedly. To reduce the likelihood of these drawbacks, a method referred to as tabu search was employed ${ }^{6}$. Tabu search alternately focuses and diversifies its search; first considering states very similar to the current state, then considering states very different. In addition, a history of the states already visited is kept so that a state, once visited, is not evaluated again.

By manipulating the parameters of the optimization process, it was possible to obtain different sensor sets, for example $s 1^{\circ}, s 2^{\circ}, s 3^{\circ}$, which return practically identical noise reductions, i.e., $J_{s l} \approx J_{s 2} \approx J_{s 3}$. A further test of the solution quality has been suggested ${ }^{7,8}$ where advantage is taken of the similarities which exist between adaptive noise control algorthms and linear regression algorithms. The variance of a regression coefficient ${ }^{9}$ is one such measure of solution quality and can be written in the context of the noise control problem as

$$
V\left(c_{k}\right)=\operatorname{diag}_{k}\left\{\sigma^{2}\left(\mathbf{H}^{c^{H}} \mathbf{H}^{c}\right)^{-1}\right\}
$$

where diag $_{k}$ denotes the $k^{\text {th }}$ diagonal, $\sigma^{2}$ is the variance of the measurement noise, and $\mathbf{H}^{c^{H}}$ is the Hermitian of $\mathbf{H}^{\mathrm{c}}$. The measurement noise is estimated by $\Sigma\left|s_{l}{ }^{\circ}\right|$ which is the error of the noise control solution ${ }^{7.8}$.
Large variances in the regression coefficients (control forces, $c_{k}$ ) can be related to dependencies (colinearities) in the transfer functions ${ }^{8,9}$. These dependencies can produce erroneous solutions, i.e., where $J_{s}$ is not minimized, and can increase the control algorithm's sensitivity to measurement noise. It is, therefore, desirable to select sensor solution sets with low control force variance.

\section{Modal Decomposition Overview}

The sound pressure field, $e_{j}$, can be decomposed into modal components to identify the acoustic modes ${ }^{3}$. The decomposition is based on:

$$
\Psi_{m}(\omega)=\iiint_{v o l} P(\mathbf{x}, \omega) \psi_{m}(\mathbf{x}) d V
$$

where the mode shapes, $\psi_{\mathrm{m}}(\mathbf{x})$, are the hardwall cavity modes and are orthogonal. $\Psi_{m}(\omega)$ is the contribution of the $m^{\text {th }}$ mode to the acoustic pressure, $\mathrm{P}(\mathrm{x}, \omega)$, as a function of frequency, $\omega . V$ is the volume of the acoustic space. For the modal decomposition, the pressure field was measured experimentally and the mode shapes were derived using NASTRAN. In reference ${ }^{3}, 40$ mode shapes were derived. The 40 modes had 7 different circular cross-section distributions as shown in figure 2. Node lines are shown in figure 2 as dashed lines. The axial modes varied as cosn $\pi / L$ where $n$ varied from 0 for a uniform axial distribution to 7 . For the modes of interest in this work, the correspondence between mode number, the circular cross-sectional distribution and the axial mode number are given in Table 1 .

A preferred actuator set is selected by inspection of the modal decompositions of the primary source and the individual actuator responses at a particular frequency. Dominant modes in the primary response are matched with dominant modes in the actuator responses. An actuator set that is well matched to the primary will return good noise reduction with little spillover. A sensor set is chosen by inspection of the pressure field. Sensors are placed at nodes and nulls to stimulate control and discourage spillover. This process becomes more difficult when the primary and actuator have mutiple strong modes. The process as a whole is heuristic and several iterations may be required to get the best results. 
Table 1. Mode number (m) vs. circular cross-section mode shape ( $Y$ ), axial mode number (n) and frequency (f)

\begin{tabular}{|c|c|c|c|}
\hline $\mathrm{m}$ & $\mathrm{Y}$ & $\mathrm{n}$ & $\mathrm{f}$ \\
\hline \hline 12 & $\mathrm{C}$ & 2 & 200.9 \\
\hline 20 & $\mathrm{D}$ & 2 & 260.2 \\
\hline 21 & $\mathrm{C}$ & 4 & 261.0 \\
\hline 22 & $\mathrm{E}$ & 2 & 265.6 \\
\hline 23 & $\mathrm{~B}$ & 5 & 270.9 \\
\hline 28 & $\mathrm{D}$ & 4 & 309.0 \\
\hline 29 & $\mathrm{E}$ & 4 & 313.6 \\
\hline
\end{tabular}

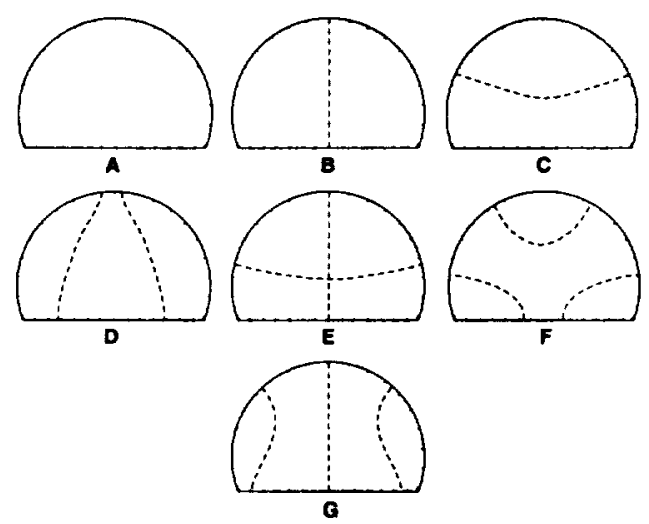

Figure 2. Circular cross-section mode shapes for cylinder interior

\section{Algorithm Optimization Overview}

The basic Time Averaged/Gradient Descent (TAGD) algorithm is straightforward. It is assumed that the mean of the squared error sensor signals (MSE) is proportional to the overall interior noise level. The controller is, therefore, designed to reduce the MSE. The controller MSE value is equivalent to the mean of the cost metric, $J_{s}$, used by the optimization procedure. It has been shown ${ }^{10}$ that the MSE is a quadratic function of the controller outputs, $c_{k}$, thus having only one minimum. Using a process of gradient descent, the TAGD controller perturbs the magnitude and phase of each actuator control signal to evaluate the gradient. The actuator signal is then moved in the direction that reduces the MSE. To keep the phase of the internal reference constant with respect to the primary noise disturbance, the controller is phase locked to the primary signal. The controller's interrupt rate is kept well above the Nyquist frequency by multiplying the phase locked signal by 8 . A distinguishing feature of the TAGD algorithm is that, unlike the filtered-X LMS algorithm $^{10}$, TAGD does not require a model of the controller subsystem.

Several parameters are available to fine tune the TAGD controller's behavior. Table 2 contains parameter values that provided dependable, but slow, noise reduction. A delay is installed between the time that an actuator is adjusted and the controller begins to sample the MSE. The delay is necessary to avoid sampling the transients which are caused by the abrupt setting of the actuator to a new value. A delay of 200 cycles (or interrupts) is equivalent to about $100 \mathrm{~ms}$ at a primary frequency of $230 \mathrm{~Hz}$. A two coefficient FIR digital filter is used to set the magnitude and phase of each controller output. The algorithm adjusts one filter weight at a time by an amount equivalent to \pm Peek $k^{*}$ MSE to determine the direction of decreasing MSE. When the correct direction is known, the controller will set the filter weight to a value of $\operatorname{sign}(P e e k)^{*}$ Step*MSE. It was found that Step must be kept less than Peek for stable operation of the controller.

Table 2. TAGD parameters

\begin{tabular}{|l|l|}
\hline Delay (cycles) & 200 \\
\hline Averages & 400 \\
\hline Peek (\%MSE) & 1.0 \\
\hline Step (\%MSE) & 0.8 \\
\hline
\end{tabular}

Given the quadratic shape of the cost function, it is possible, in theory, to enhance the performance of the basic TAGD. A parabola can be fit to a slice through the surface, the slice being defined by 3 or more data points. The minimum of the parabola can then be computed and the control set to the projected minimum. This takes more computation for a given step, but this single step should produce very good results. In practice, good single step performance is not observed. The modified algorithm searches for the surface minimum as before yet with the added burden of increased computation. This poor performance can be attributed to the dependencies which exist between the actuators that alters the topology of the MSE function for one actuator as another is stepped. This shifting is illustrated in figures $3 a$ and $3 b$.

Figure 3a is a surface plot of the MSE of one actuator over a small range of the two controller filter weights. Figure $3 \mathrm{~b}$ is the MSE surface for the same actuator, over the same range of filter weights when a second actuator has been turned on. The MSE relationship for the first actuator has changed 


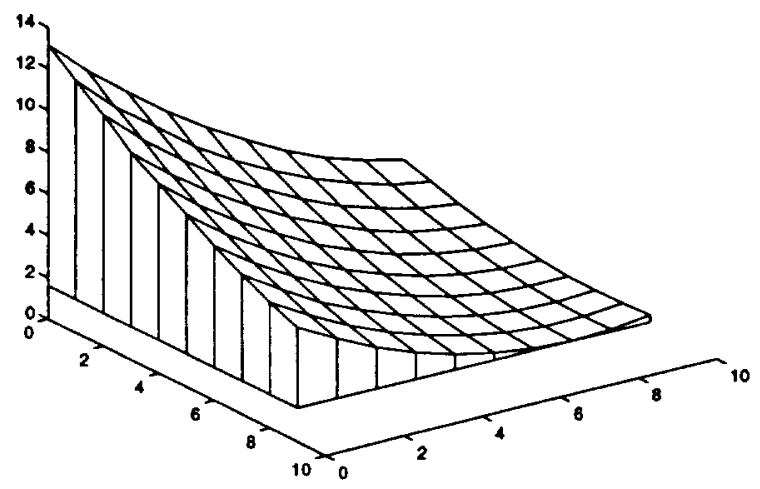

Figure 3a. MSE surface of one actuator

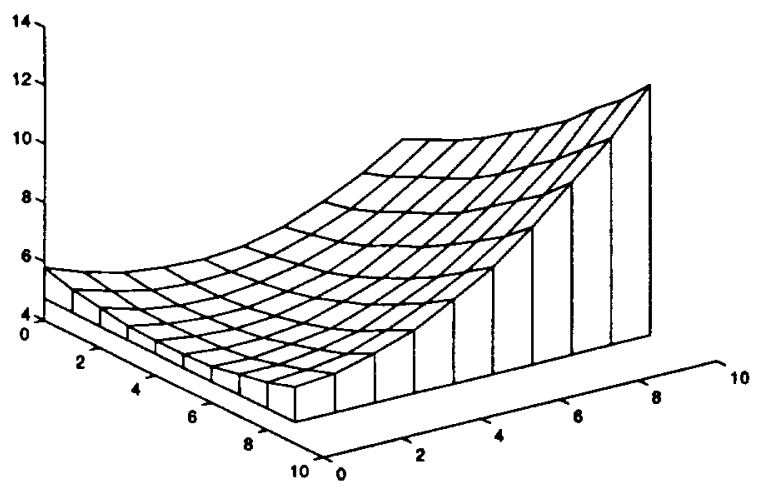

Figure 3b. MSE surface of actuator with interference

dramatically due to the influence of the second actuator. This phenomena can cause poor convergence and erratic performance. The observed shifting demostrates that the actuators are not linearly independent.

Brincipal Components. The actuator array can be decomposed into a matrix of independent (orthogonal) principal components using singular value decomposition ${ }^{11,12}$. In effect, $n$ dependent actuators are transformed into $n$ independent $n x l$ vectors. To design the control algorithm in terms of principal components, equation (4) is first written in matrix form and without the primary disturbance .

$$
\mathbf{s}=\mathbf{H c}
$$

Where $s$ is a $N_{s} x 1$ vector of sensor readings, $c$ is a $N_{c} \times 1$ vector and $H$ is the $\mathrm{N}_{s} \mathrm{xN}_{\mathrm{c}}$ transfer function matrix. For the single frequency case, the elements of $\mathbf{H}$ are complex values. Applying singular value decomposition, equation (8) becomes,

$$
\mathbf{s}=\mathbf{U S V}^{H} \mathbf{c}
$$

where $S$ (a $\mathrm{N}_{8} \mathrm{xN}_{\mathrm{a}}$ matrix) contains real singular values on its diagonal and both $U\left(a N_{8} \times N_{8}\right.$ matrix) and $\mathrm{V}$ (a $\mathrm{N}_{\mathrm{c}} \times \mathrm{N}_{\mathrm{c}}$ matrix) are unitary matrices, i.e., $\mathrm{UU}^{\mathrm{H}}=1$. Equation (9) is rearranged to obtain

$$
\mathbf{s}_{p c}=\mathbf{S} \mathbf{c}_{p c}
$$

where $s_{p c}=U^{H} s$ and $c_{p c}=V^{h} c$. The norm of $s_{p c}$ is the same as the norm of $s$, as in equation (5), owing to the unitary nature of $\mathbf{U}$.

The next section describes the experimental procedure employed to evaluate the optimization methodologies.

\section{Experimental Procedure}

An array of 8 piezoceramic patches installed on a trim panel in the composite fuselage model served as a test case for the optimization methodology. In general, the procedure followed the optimization methodology as outlined above. Broad band transfer function data in the range of $150 \mathrm{~Hz}$ to $650 \mathrm{~Hz}$ was obtained for the primary disturbance and each of the actuators by sweeping a microphone boom throughout the interior of the model. A total of 462 transfer functions was obtained from the sweep. In addition, structural data was obtained from a set of accelerometers installed on the trim panels. The acoustic and structural data were used to select 3 frequencies of interest. These were $210 \mathrm{~Hz}$ (strong acoustic mode, strong structural mode), $230 \mathrm{~Hz}$ (weak acoustic mode, strong structural mode) and $275 \mathrm{~Hz}$ (strong acoustic mode, weak structural mode).

The optimization procedure was then used to select the best 4 out of 8 actuators and 8 out of 462 microphone positions for each of the 3 frequencies. The performance of the selected actuators and sensors was tested by configuring the model accordingly and using the TAGD controller to minimize the mean square error of the sensor microphones. The interior of the cylinder was then surveyed to obtain overall SPL. Noise reduction for the interior is determined with respect to a baseline, no-control case. These results are compared with an architectural configuration derived using the modal decomposition technique described by Lyle $^{3}$. 
The following sections describe the composite model test facility, details of the optimization process and the controller design.

The Composite Fuselage Model. The composite fuselage model is shown in figure 4 . The cylinder is $3.6 \mathrm{~m}$ long and $1.68 \mathrm{~m}$ in diameter. The outer shell is a 9 layer filament wound graphite epoxy composite. Total skin thickness is $1.7 \mathrm{~mm}$. The cylinder takes on fuselage attributes in that it is stiffened with composite stringers and ring frames. A plywood floor is supported on the ring frames $0.544 \mathrm{~m}$ above the bottom of the cylinder. To complete the fuselage model, 3 inner trim panel sections are hard mounted to the ring frames. The trim panels have a $6.35 \mathrm{~mm}$ honeycomb core with 0.64

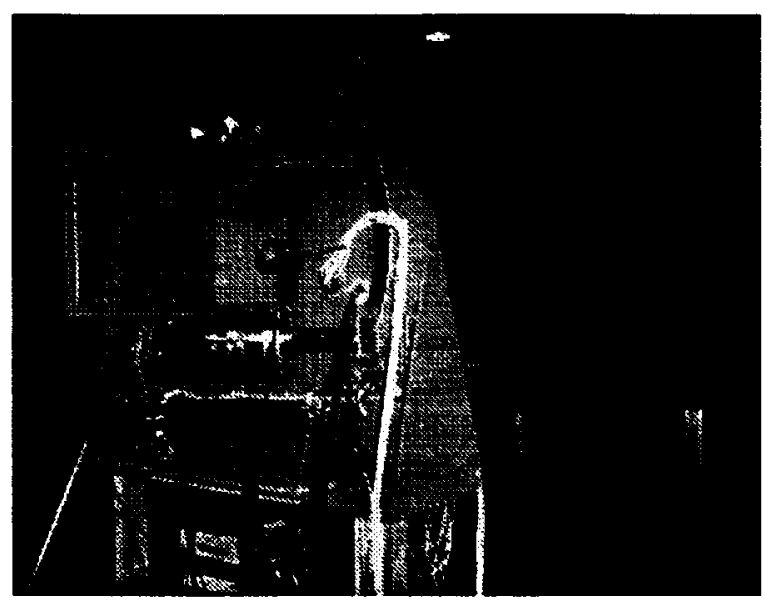

Figure 4. The composite model fuselage

mm graphite epoxy laminate sheet bounded to either side of the core. Refer to Lyle ${ }^{3}$ for a more detailed description of the model.

Measurement Instrumentation. The measurement instrumentation consisted of the primary source, microphone boom traverse and related acquisition equipment. The primary source was a 100 watt, baffled, electrodynamic loudspeaker. The speaker was mounted at a radial position of $1.1 \mathrm{~m}$ and angle of $-90^{\circ}$ relative to the cylinder's centerline, $0.3 \mathrm{~m}$ from the exterior sidewall. In the interior, six equally spaced $12.7 \mathrm{~mm}$ microphones were mounted on a boom between the radial distances of $r=0.13 \mathrm{~m}$ and $r=0.73 \mathrm{~m}$. The boom could be swept from azimuthal position $\theta=-108^{\circ}$ to $\theta=108^{\circ}$. The axial traversing mechanism could translate the boom from $x=0.356 \mathrm{~m}$ to $x=3.59 \mathrm{~m}$. See figure 5 for a sketch of the layout.

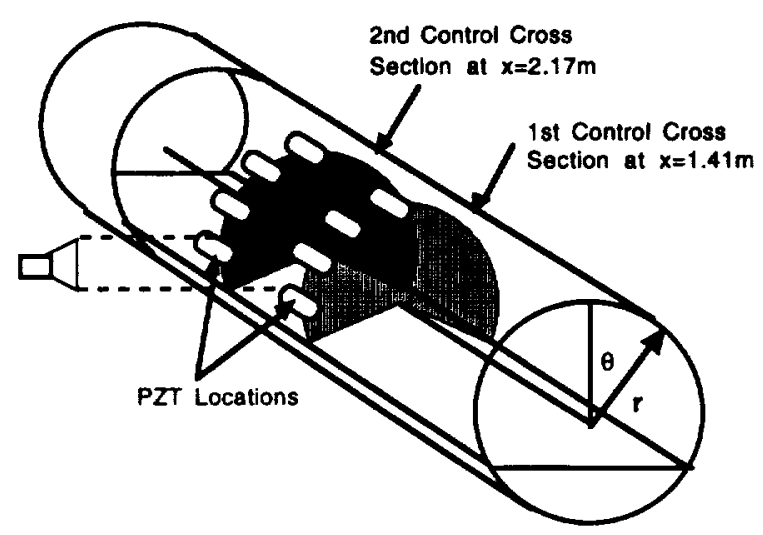

Figure 5. Sketch of instrumentation layout

Data were taken at 11 azimuthal positions and 7 axial locations for a total of 462 points $(7 \times 11 \times 6)$ within the interior cylinder volume. The azimuthal positions were $\theta \in\left\{ \pm 108^{\circ}, \pm 84^{\circ}, \pm 60^{\circ}, \pm 36^{\circ}, \pm 12^{\circ}, 0^{\circ}\right\}$. The axial locations were $x \in\{0.36 \mathrm{~m}, 0.88 \mathrm{~m}, 1.49 \mathrm{~m}, 1.79 \mathrm{~m}$, $2.1 \mathrm{~m}, 2.71 \mathrm{~m}, 3.23 \mathrm{~m}$. A stationary reference microphone was mounted at $\left(x=0, r=1.0 \mathrm{~m}, \theta=108^{\circ}\right)$ as an acoustic reference for diagnostic purpose. These 7 channels plus the primary signal which drove the speaker were acquired at each position in the matrix. The coherence and transfer function with respect to the primary signal were computed and monitored in real time during each survey.

\section{Controller Instrumentation. Controller} instrumentation consisted of the actuator array, 8 error sensors and the controller digital signal processor. The actuators were lead zirconate titanate (PZT5)

piezoceramic patches measuring $3.81 \mathrm{~cm}$ by $7.62 \mathrm{~cm}$. The patches were machined to match the curvature of the trim panel and were bonded to its outer surface. The patches were installed in pairs, 16 patches yielding 8 actuators. The actuators were mounted at 2 axial locations, 4 actuators at $x=1.41 \mathrm{~m}$ and 4 actuators at $x=2.17 \mathrm{~m}$. These axial locations are on the center trim panel and equidistant from the midpoint. Each pair of patches were spaced $6.35 \mathrm{~mm}$ apart and centered at angles $\theta \in\left\{0^{\circ},-30^{\circ},-60^{\circ},-90^{\circ}\right\}$. The actuator locations are shown in figure 5 . The error sensors were $4.8 \mathrm{~mm}$ electret-condenser microphones. They were mounted on stands and positioned in the interior according to the optimization specification. The control algorithm executed in a PC based digital signal processor (TMS320C30). The controller had the capability to acquire 8 channels and output 6 channels. See figure 6 for a diagram of the controller. 


\section{Architecture Optimization}

Based on measured transfer functions, preliminary actuator optimization runs were made selecting the best sets of 3,4 and 5 actuators for 8 frequencies. Increasing the number of actuators from 3 to 4 actuators improved the noise reduction on average $0.65 \mathrm{~dB}$, but, increasing

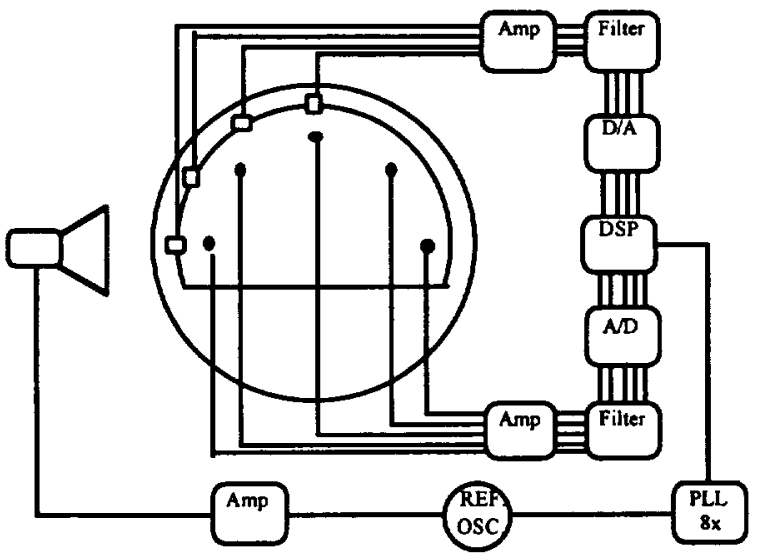

Figure 6. Controller instrumentation

the number from 4 to 5 actuators improved the noise reduction only $0.28 \mathrm{~dB}$. Based on these results, the best of 4 out of 8 actuators were chosen at each frequency of interest $(210 \mathrm{HZ}, 230 \mathrm{~Hz}, 275 \mathrm{~Hz})$.

Results from the sensor optimization showed it was possible to obtain sensor solutions where the derived control forces had high variances (see discussion of variance in the Architecture Optimization Overview section). It was believed that those sensor solutions which caused high control force variances would be hard to control. To test this hypothesis, sensor solutions with both high and low actuator force variances were calculated for the 3 frequencies of interest. For each case, the best 8 out of 462 sensor locations were found for each case.

The potential benefit of actuator/sensor optimization can be seen in figures $7 \mathrm{a}$ through $7 \mathrm{c}$ where the predicted noise reduction for 1000 samples of 8 randomly selected sensors is plotted in a histogram for both the best case and worst case set of 4 actuators (the best case actuators return the greatest noise reduction measured at all 462 microphone locations, the worst case microphones return the least). Observe that the best case distributions are similar for the three frequencies. The best case distributions indicate that fair noise reduction can be obtained with $50 \%$ of the randomly selected
Random Sensor Locations at $275 \mathrm{~Hz}$ GBeed Caes Worst Case

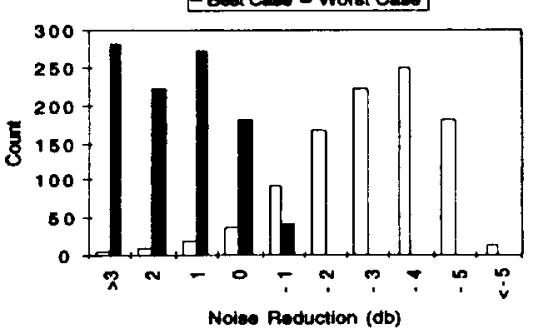

Figure 7a. Noise reduction at $275 \mathrm{~Hz}$

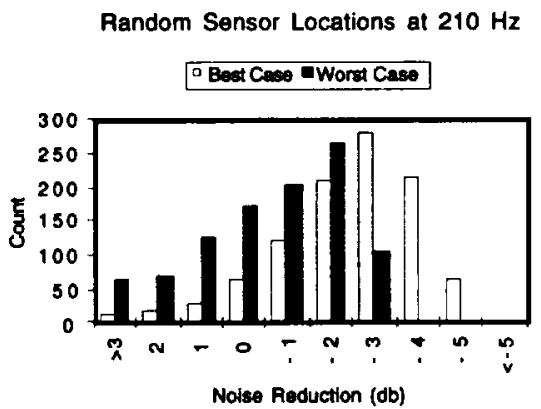

Figure $7 \mathrm{~b}$. Noise reduction at $210 \mathrm{~Hz}$

Random Locations at $230 \mathrm{~Hz}$ o Beet Case aWorst Case

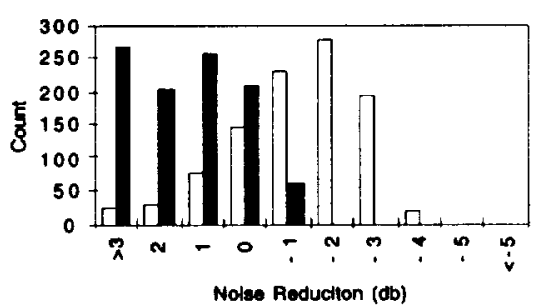

Figure 7c. Noise Reduction at $230 \mathrm{~Hz}$

sensor locations. The distributions also indicate that that fewer than $5 \%$ of the randomly selected sensor locations return the greatest reduction. The maximum reduction for $210 \mathrm{~Hz}$ and $275 \mathrm{~Hz}$ was approximately $-5.5 \mathrm{~dB}$. The maximum reduction for $230 \mathrm{~Hz}$ was $-3.8 \mathrm{~dB}$. Notice also that the shape of the distributions change dramatically for the worst case actuators at $275 \mathrm{~Hz}$ and $230 \mathrm{~Hz}$, yet remains basically the same for $210 \mathrm{~Hz}$. This may be due to the single acoustic mode which dominates at $210 \mathrm{~Hz}$. Both $230 \mathrm{~Hz}$ and $275 \mathrm{~Hz}$ have multiple modes of approximately equal amplitude. These distributions seem to indicate that it should be easier to get fair performance from a noise field with a single dominant mode vs. one with multiple, 
competing, modes. The modal decomposition of the data is discussed in more detail in the following.

Results. Tables 3 through 5 show the results obtained for the 3 test frequencies. At each frequency the 4 criteria for locating the error microphone sources were, control force-high variance (HV), control forcelow variance (LV), worst case actuator set (WC) and modal decomposition (MD). For $230 \mathrm{~Hz}$, the modal response was too weak to support the decomposition analysis making the actuator selection almost random. The microphone locations were selected by inspection of the primary response field (PR). The sum of the variance values are printed along side their respective symbols. Note that the $275 \mathrm{~Hz} \mathrm{HV}$ case has a low variance when compared with the variances of $210 \mathrm{~Hz}$ and $230 \mathrm{~Hz} \mathrm{HV}$ cases. For the error microphone configurations both the predicted noise reduction and reduction actually obtained with TAGD are shown. The TAGD results are given in terms of both the relative amount of noise reduction and the actual controlled SPL/uncontrolled SPL values.

Table 3. Optimization results at $210 \mathrm{~Hz}$

\begin{tabular}{|c||c|c|c|}
\hline \multicolumn{4}{|c|}{ Strong Acoustic/ Strong Structural } \\
\hline \multirow{2}{*}{$\begin{array}{c}\text { Error Mic. } \\
\text { Criteria }\end{array}$} & \multicolumn{3}{c|}{ Noise Reduction (dB) } \\
\cline { 2 - 4 } & Predicted & \multicolumn{2}{c|}{ TAGD } \\
\hline HV(47.2) & -5.3 & -2.5 & $73.7 / 76.2$ \\
\hline LV(13.2) & -5.3 & -4.4 & $72.2 / 76.6$ \\
\hline WC & -3.5 & -3.1 & $73.5 / 76.6$ \\
\hline MD & NA & -2.1 & $72.3 / 74.4$ \\
\hline
\end{tabular}

Table 4. Optimization results at $275 \mathrm{~Hz}$ Strong Acoustic/ Weak Structural

\begin{tabular}{|c||c|c|c|}
\hline \multicolumn{3}{|c|}{ Strong Acoustic/ Weak Structural } \\
\hline \multirow{2}{*}{$\begin{array}{c}\text { Error Mic. } \\
\text { Criteria }\end{array}$} & \multicolumn{3}{c|}{ Noise Reduction (dB) } \\
\cline { 2 - 4 } & Predicted & \multicolumn{3}{c|}{ TAGD } \\
\hline HV(6.9) & -5.7 & -6.4 & $74.1 / 80.5$ \\
\hline LV(2.6) & -5.7 & -3.9 & $75.1 / 79.0$ \\
\hline WC & -0.5 & -0.4 & $78.6 / 79.0$ \\
\hline MD & NA & -2.7 & $76.4 / 79.1$ \\
\hline
\end{tabular}

The sensor/actuator arrays selected through combinatorial optimization (LV cases) returned greater noise reduction than the modal decomposition cases (MD). However, at $210 \mathrm{~Hz}$ where the modal spectrum is dominated by a single acoustic mode (12), both the $\mathrm{LV}$ and MD cases reduced the noise to approximately the same level $(\sim 72.2 \mathrm{~dB})$. The major difference in the noise reduction is due to differences in the uncontrolled SPL. The discrepancy in uncontrolled SPL is thought to be caused by changes in environmental conditions which modified the structural/acoustic response of the cylinder. Small changes in temperature, for example, may significantly alter the structural/acoustic coupling. The MD case used 2 actuators and 6 microphones and is a reproduction of the $210 \mathrm{~Hz}$ case reported in reference 3.

Table 5. Optimization results at $230 \mathrm{~Hz}$

\begin{tabular}{|c||c|c|c|}
\hline \multicolumn{4}{|c|}{ Weak Acoustic/ Strong Structural } \\
\hline \multirow{2}{*}{$\begin{array}{c}\text { Error Mic. } \\
\text { Criteria }\end{array}$} & \multicolumn{3}{c|}{ Noise Reduction (dB) } \\
\cline { 2 - 4 } & Predicted & \multicolumn{2}{c|}{ TAGD } \\
\hline HV(22.6) & -3.7 & -0.1 & $72.6 / 72.7$ \\
\hline LV(8.6) & -3.8 & -2.5 & $70.7 / 73.5$ \\
\hline WC & -0.5 & +0.2 & $73.7 / 73.5$ \\
\hline PR & NA & -1.2 & $70.8 / 72.0$ \\
\hline
\end{tabular}

The $275 \mathrm{~Hz}$ acoustic response is dominated by several modes $(20,21,22,23,28$ and 29). These modes span 4 cross-sectional shapes (see table 1 and figure 2). Unlike $210 \mathrm{~Hz}$, the uncontrolled SPL remained relatively constant. This is thought to be due to the large number of acoustic modes with which the structure can couple. The LV case had better noise reduction than the MD case indicating that the complexity of the modal response makes it more difficult to select actuators using modal decomposition.

In general the results indicate that selecting the right combination of actuators and sensors significantly improves noise control performance. The LV, MD and PR cases outperformed the WC case. The LV cases returned better noise reduction than the HV cases demonstrating that microphone arrays that have corresponding control forces of low variance are easier to control. The relative performance of the LV and WC responses are well modeled by the distributions in figures 7a through 7c. A large difference exists between the $\mathrm{LV}$ and $\mathrm{WC}$ cases for $230 \mathrm{~Hz}$ and $275 \mathrm{~Hz}$ while the difference between the $210 \mathrm{~Hz} \mathrm{LV}$ and WC cases is about $1 \mathrm{~dB}$.

\section{Principal Component Control Algorithm}

The TAGD control algorithm was redesigned to use the principal components of the actuator and sensor arrays. The algorithm perturbed one element of the principal component vector, $c_{p c}$, at a time, then transformed the result into the physical actuator settings using $\mathbf{c}=\mathbf{V} \mathbf{c}_{\mathrm{pc}}$. The subsequent sensor readings, $\mathbf{s}$, were then transformed into $s_{p c}$ using $s_{p c}=U^{H} s$. The component of $s_{p c}$ corresponding to the originally perturbed component of $c_{p c}$ was used to evaluate the 
effect of the change. It should be noted that only the first $N_{c}$ terms of $s_{p c}$ are controllable, i.e., any cost value in terms other than the first $\mathbf{N}_{\mathrm{c}}$ cannot be reduced. For these tests, $\mathrm{N}_{\mathrm{c}}=4$ and $\mathrm{N}_{\mathrm{s}}=8$.

Results. The principal component TAGD controller, PC-TAGD, performed as theory predicted. Individual components could be perturbed without effecting the others. Figures $8 \mathrm{a}$ and $8 \mathrm{~b}$ show the error surface of a principal component in the absence and in the presence of a second principal component. Notice that in contrast to the single actuator case (figures $3 \mathrm{a}$ and $3 \mathrm{~b}$ ), the shape of the principal component error surface has not changed at all. The curve in $8 \mathrm{~b}$ is elevated relative to figure $8 \mathrm{a}$ due to the presence of the added noise power from the second principal component.

The PC-TAGD controller was run at all 3 frequencies for the low variance configuration. The performance of PC-TAGD relative to the basic TAGD is shown in Table 6. In general PC-TAGD performed

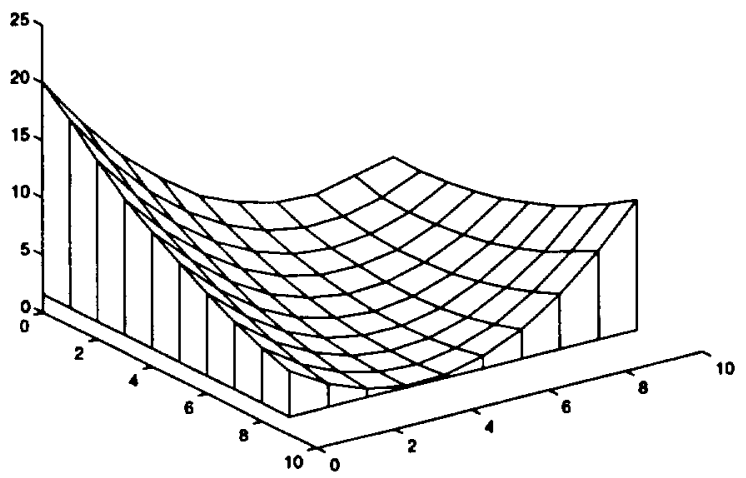

Figure 8a. Single principal component

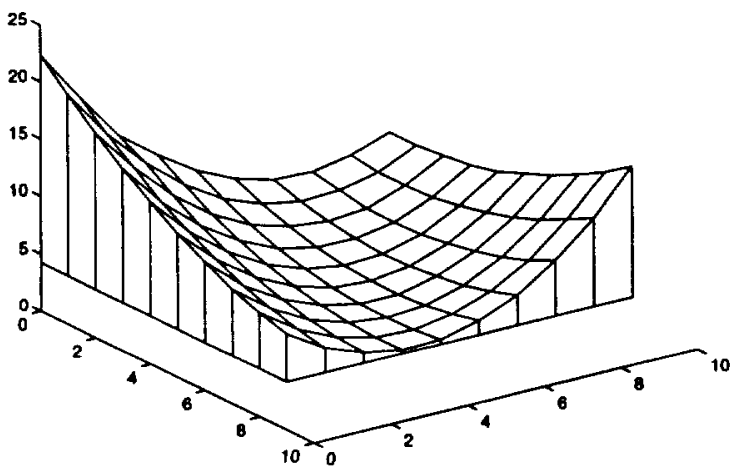

Figure 8b. Principal component with interference as well as TAGD. The $210 \mathrm{~Hz}$ case stands out as an exception. The reduction in PC-TAGD performance $(-4.4$ vs. -2.6$)$ for the $210 \mathrm{~Hz} \mathrm{LV}$ case may be due to excess effort to reduce a principal component which is poorly suited to the control problem ${ }^{13}$. The excellent partitioning provided by the principal components in effect raises each component to the same level of observability, regardless of the component's relative ability to control the noise field.

Table 6. PC-TAGD performance

\begin{tabular}{|c|c|c|c|c|}
\hline \multirow{2}{*}{ Freq. } & \multicolumn{4}{|c|}{ Noise Reduction (dB) } \\
\cline { 2 - 5 } & \multicolumn{2}{|c|}{ TAGD } & \multicolumn{2}{|c|}{ PC-TAGD } \\
\hline 210 & -4.4 & $72.2 / 76.6$ & -2.6 & $73.4 / 76.0$ \\
\hline 230 & -2.5 & $70.7 / 73.5$ & -2.4 & $70.9 / 73.3$ \\
\hline 275 & -3.9 & $75.1 / 79.0$ & -4.1 & $74.8 / 78.9$ \\
\hline
\end{tabular}

This phenomenon is illustrated in figure 9 where a plot of SPL vs. relative MSE is shown for two actuator placements, an optimum case and a random case. The SPL decreases with MSE for the optimum case as expected. The increase in SPL for the random case occurred as the controller tried to reduce the MSE of the last principal component. This demonstrates that the control effort required to reduce the error of the last principal component caused spillover of control energy and an increase in the global SPL. An optimumly selected actuator/sensor set may reduce the chance that a principal component is ill-behaved. However, as the $210 \mathrm{~Hz}$ case demonstrates, using an optimally actuator/sensor set may not be sufficient to guarantee the effectiveness of all principal components.

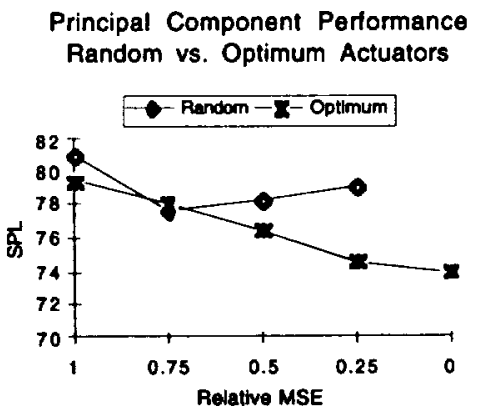

Figure 9. Two cases of principal component performance 


\section{Concluding Remarks}

Two aspects of optimization have been explored. One, actuator/sensor architecture optimization, deals with the number and placement of the actuators and sensors. The other, algorithm optimization, is concerned with how to best design a control algorithm to work with arrays of actuators and sensors. It has been found that for frequencies where multiple acoustic modes are present, that it is necessary to perform some type of optimization of the number and placement of the actuators and sensors. It is also evident that modal decomposition methods break down when the modal spectrum gets too complex. The combinatorial optimization technique used herein performed well in all cases. A disadvantage of the technique is that the transfer functions of all the actuator locations under consideration must be produced either experimentally or analytically. It is unlikely that a transfer function of sufficient quality can be produced analytically. The alternative experimental approach has limits in the number of actuator placements that can be tested. For example, a comprehensive study of a $10 \times 10$ area would require that 100 actuators be attached to the surface. If this were done, questions concerning the affect of the large number of actuators on the structure's response would arise. The means of performing this type of comprehensive test needs to be developed before this optimization technique can be used to its fullest potential.

If arrays of actuators and sensors are to be controlled, then a principal component based controller has the distinct advantage of creating a virtual set of independent actuators and sensors. With principal component control in place other optimization techniques which require a stationary cost function can be used. The increased overhead of computing optimized solutions can be addressed by operating the principal component controller in parallel. The principal component algorithm depends on the actuator transfer functions. Thus, the PC-TAGD controller may require some kind of on-line system identification to function properly. The dependency of principal component based control on the accuracy of its transfer function models remains to be evaluated.

\section{References}

1. Fuller, C.R., Snyder, S., Hansen, C. and Silcox, R.J." "Active Control of Interior Noise in Model Aircraft Fuselages using Piezoceramic Actuators", AIAA Journal, Vol 30, no 11, pp 2613-2617, November 1992.

2. Silcox, R.J., Lefebvre, S., Metcalf, V.L., Beyer, T.B. and Fuller, C.R.: "Evaluation of Piezoceramic Actuators for Control of Aircraft Interior Noise", Proceedings of DGLR/AIAA 14th Aeroacoustics Conference, vol 2, pp 542-551, May 1992.

3. Lyle, K.H. and Silcox, R.J.: "A Study of Active Trim Panels for Noise Reduction in an Aircraft Fuselage", paper no. 951179 in SAE General, Corporate and Regional Aviation Meeting and Exposition, May 1995.

4. Silcox, R.J., Fuller, C.R., and Lester, H.C.:"Mechanisms of Active Control in Cylindrical Fuselage Structures", AIAA Journal, vol. 28, no. 8, pp. 1397-1404, August 1990.

5. Padula, S.L. and Kincaid, R.K.: "Aerospace Applications of Integer and Combinatorial Optimization", NASA Technical Memorandum 110210, October 1995.

6. Glover, F.: "Tabu Search: A Tutorial", Interfaces, vol. 20, pp. 74-94, 1990.

7. Snyder, S.D. and Hansen, C.H.: "Using Multiple Regression to Optimize Active Noise Control System Design", Journal of Sound and Vibration, vol. 148, no. 3, pp. 537-542, 1991.

8. Ruckman, C.E. and Fuller, C.R.: :A Regression Approach for Simulating Feedforward Active Noise Control", Journal of Acoustical Society of America, vol. 97, no. 5, pp.2901-2918, 1995.

9. Draper, N.R. and Smith, H.: "Applied Regression Analysis", John Wiley and Sons, 2nd edition, 1981.

10. Elliot, S.J., Stothers, I.M., ans Nelson, P.A.: "A Multiple Error LMS Algorithm and its Application to the Active Control of Sound and Vibration", IEEE Transactions on Acoustics, Speech and Signal Processing, pp. 1423-1434, 1987.

11. Strang, G.: "Linear Algebra and Its Applications", Third Edition, Harcout Brace Jovanovich, 1988.

12. Jolliffe, I.T.: "Principal Component Analysis", Springer-Verlag, 1986.

13. Elliot, S.J. and Boucher, C.C.: "The Behavior of a Multiple Channel Active Control System. IEEE Transcations on Signal Processing, vol .40, no. 5, May 1992. 



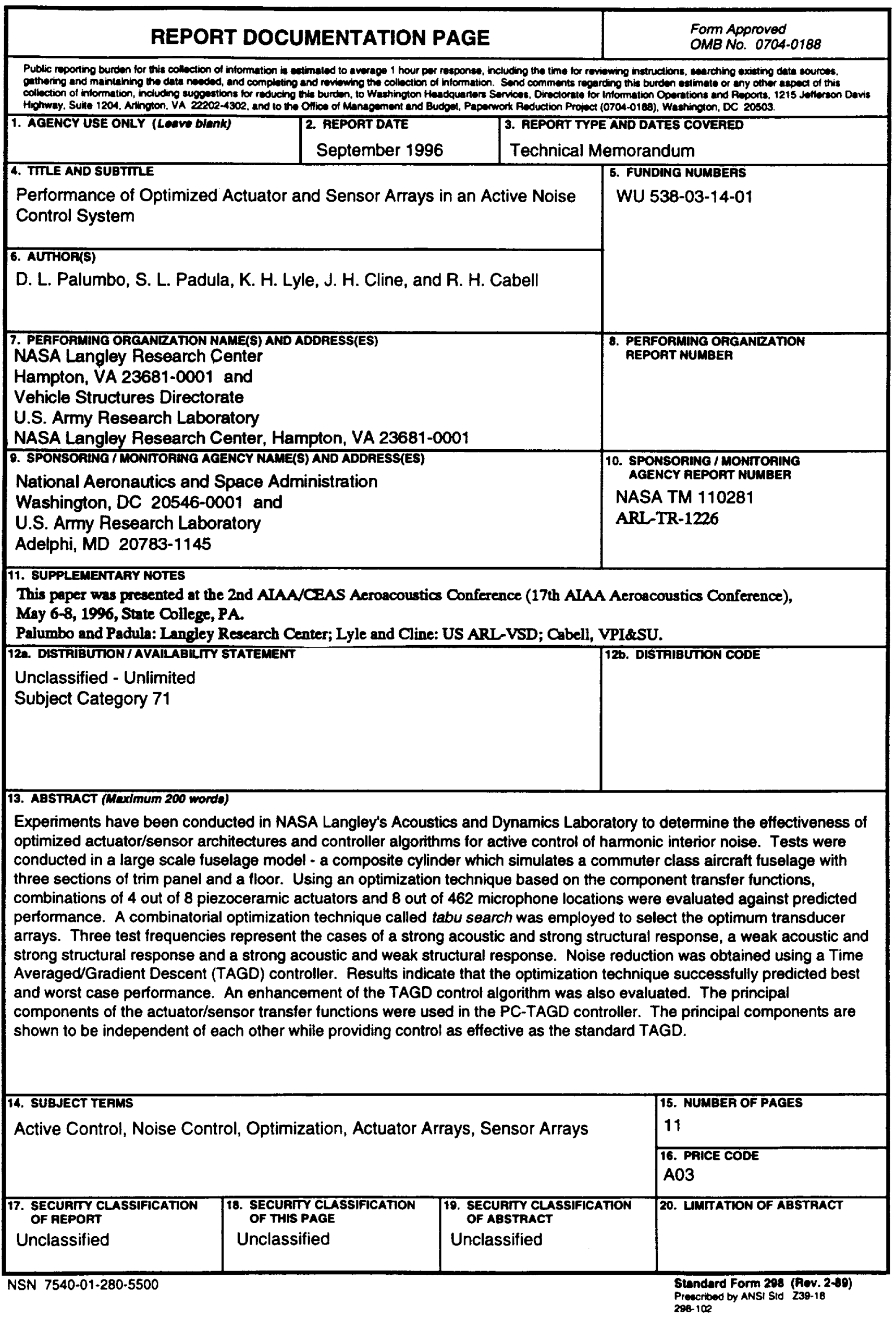

